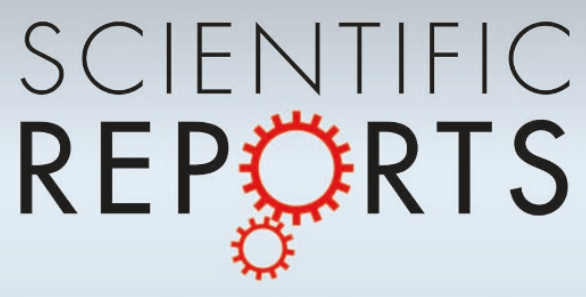

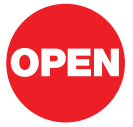

SUBJECT AREAS:

NANOPARTICLES

METAMATERIALS

ULTRAFAST PHOTONICS

SENSORS AND BIOSENSORS

Received

3 January 2013

Accepted

8 April 2013

Published

3 May 2013

Correspondence and requests for materials should be addressed to W.L.Z. (weili.zhang@ okstate.edu)

\section{Tailoring terahertz plasmons with silver nanorod arrays}

\author{
Wei Cao' ${ }^{1}$ Chunyuan Song ${ }^{2}$, Thomas E. Lanier ${ }^{2}$, Ranjan Singh ${ }^{3}$, John F. O'Hara' ${ }^{1}$, William M. Dennis ${ }^{2}$, \\ Yiping Zhao' \& Weili Zhang'
} 'School of Electrical and Computer Engineering, Oklahoma State University, Stillwater, Oklahoma 74078, USA, ${ }^{2}$ Department of
Physics and Astronomy, University of Georgia, Athens, Georgia 30602, USA, ${ }^{3}$ AOT-HPE, Los Alamos National Laboratory, New
Mexico 87545, USA.

Plasmonic materials that strongly interact with light are ideal candidates for designing subwavelength photonic devices. We report on direct coupling of terahertz waves in metallic nanorods by observing the resonant transmission of surface plasmon polariton waves through lithographically patterned films of silver nanorod (100 $\mathrm{nm}$ in diameter) micro-hole arrays. The best enhancement in surface plasmon resonant transmission is obtained when the nanorods are perfectly aligned with the electric field direction of the linearly polarized terahertz wave. This unique polarization-dependent propagation of surface plasmons in structures fabricated from nanorod films offers promising device applications. We conclude that the anisotropy of nanoscale metallic rod arrays imparts a material anisotropy relevant at the microscale that may be utilized for the fabrication of plasmonic and metamaterial based devices for operation at terahertz frequencies.

der erahertz (THz) plasmonic metamaterials is an exciting and timely new research area. THz radiation, as defined in the frequency range of $0.1-10 \mathrm{THz}$, has a multitude of desirable attributes. These include a nonionizing nature, good transmission through many optically opaque materials, and the ability to probe signatures of biochemical molecules and illegal drugs. Thus, $\mathrm{THz}$ radiation has extensive applications in a broad range of disciplines, particularly sensing, imaging, and spectroscopy ${ }^{1-16}$. Both electronics and optics are pushing their boundaries into this far-infrared region, thereby offering the promise of future wide-scale development. A difficult hurdle for both approaches is the lack of natural materials that respond favorably to $\mathrm{THz}$ radiation. $\mathrm{THz}$ plasmonics and metamaterials are promising because they can be specifically designed at will to respond to $\mathrm{THz}$ radiation in ways that naturally occurring materials cannot, and could therefore impact broad application areas including civilian and military radar systems, local covert communications, optoelectronics, and THz imaging.

Currently, two challenges in $\mathrm{THz}$ plasmonics and metamaterials include: (1) finding novel approaches to transition to next-generation $\mathrm{THz}$ devices, and (2) overcoming loss mechanisms. The latter presents a fundamental barrier in the available conductivity of metals ${ }^{17}$. In terms of the former, remarkable progress has been made in THz generation and detection in the last two decades, but there is a great demand for basic components necessary to manipulate $\mathrm{THz}$ waves. $\mathrm{THz}$ metamaterials are a timely invention that offers promise here. $\mathrm{THz}$ metamaterial based negative index of refraction ${ }^{18}$, modulators ${ }^{19}$, absorbers ${ }^{20}$, invisibility cloaking ${ }^{21,22}$, proof-ofconcept demonstrations of thin film sensing ${ }^{23-25}$, antennas ${ }^{26-29}$, and unique optical activities ${ }^{30}$, all herald the development of next-generation $\mathrm{THz}$ devices for a broad area of applications. Many of these demonstrations are based on hybrid metamaterial concepts, where tuned electromagnetic properties of the constituents (e.g. silicon) convey additional functionality to the macroscopic metamaterial ${ }^{31}$. However, nanostructuring the constituents, to modify their bulk electromagnetic response before inclusion into $\mathrm{THz}$ plasmonic metamaterials, are unexplored. Recent developments in nanofabrication have opened new opportunities to fabricate metallic nanostructures with tunable porosity and alignment, and can be integrated into conventional micro-fabrication process. Engineered nanostructures with controlled porosity and morphology could systematically tune the dielectric and polarization properties of metals in the $\mathrm{THz}$ regime. The combination of the material control on multiple size scales could be a key enabler. Combining nanostructured thin films with microstructured plasmonic metamaterials will give us a new opportunity to design and explore novel $\mathrm{THz}$ devices with unique functionality.

\section{Results}

Design and measurements of the nanorod plasmonic arrays. In this article we report a study of the $\mathrm{THz}$ response of well-aligned silver nanorod array films and a THz surface plasmon (SP) resonant device ${ }^{32}$ that has 
been lithographically patterned using these silver nanorod films. Our results demonstrate that anisotropic thin metallic nanorod structures can be used to tune the $\mathrm{THz}$ response and can be another important strategy to design $\mathrm{THz}$ plasmonic metamaterial based devices.

Aligned and tilted silver nanorod (AgNR) arrays were fabricated directly on silicon substrates by oblique angle deposition (OAD) method in a custom-designed electron-beam evaporation system ${ }^{33,34}$. During the deposition, the Ag vapor flux was incident onto the silicon substrates at an angle of $86^{\circ}$, resulting in nanorods of $1 \mu \mathrm{m}$ in length, $100 \mathrm{~nm}$ in diameter, and a tilt angle of $72^{\circ}$ with respect to the surface normal ${ }^{33,34}$. Figure 1 a shows a representative top view image of the Ag nanorod arrays obtained by a scanning electron microscope (SEM, FEI Inspect F). The length, tilt angle, and porosity of the AgNRs can be controlled by the deposition rate, duration, and vapor incident angle, which result in tunable optical properties ${ }^{35}$. A terahertz time-domain spectroscopy (THz-TDS) system was employed to characterize the nanorod samples ${ }^{4,36}$. Angle dependent transmission measurement was carried out to characterize the polarization properties of the unpatterned AgNR array. After each timedomain measurement, the sample was rotated with respect to the normal of its surface at an interval of $10^{\circ}$.

It was found that the $\mathrm{THz}$ properties of the nanorod arrays are tunable; in particular, the anisotropic arrangement of the AgNR arrays induces a polarization dependence in the transmission of terahertz waves. Figures $2 \mathrm{a}$ and $2 \mathrm{~b}$ show the transmitted timedomain pulses and the corresponding Fourier-transformed amplitude spectra of an AgNR array oriented at $0^{\circ}$ and $90^{\circ}$, respectively. Here, $0^{\circ}$ and $90^{\circ}$ represent the polarization of the $\mathrm{THz}$ electric field parallel and perpendicular to the direction of the long axis of the nanorod's projection onto the silicon substrates. As shown in Fig. 2b, the unique anisotropic structure of the AgNR arrays resulted in a $4: 1$
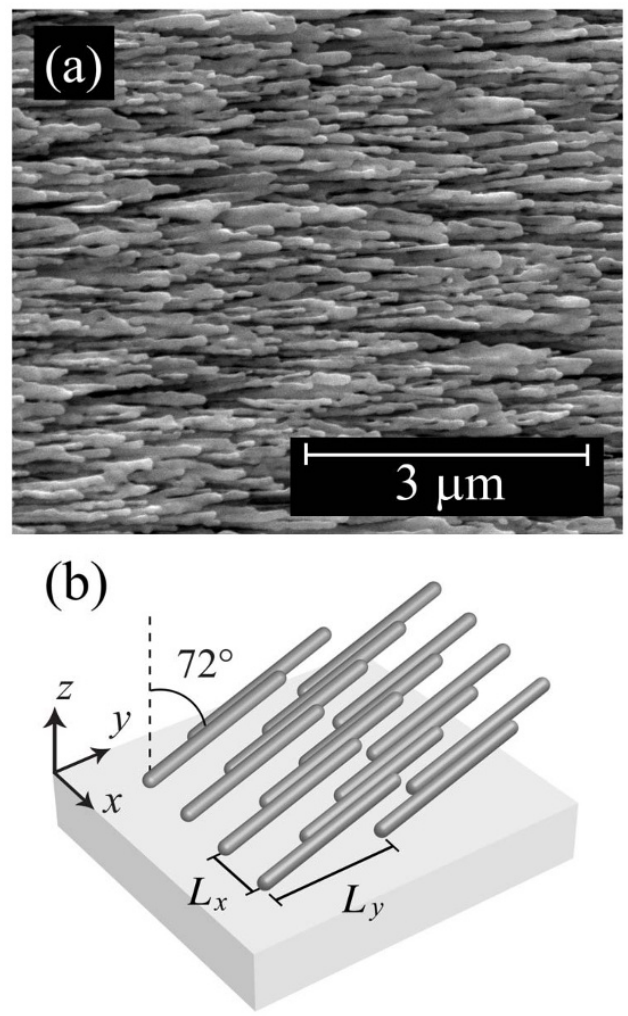

Figure 1 Illustration of morphology and dimensions of Ag nanorods. (a) SEM image of the Ag nanorods deposited on a silicon wafer.

(b) Staggered base morphology derived from the SEM image. The rods have a diameter $100 \mathrm{~nm}$, length $1 \mu \mathrm{m}$, and are oriented at a $72^{\circ}$ angle with the substrate normal. $L_{x}=250 \mathrm{~nm}, L_{y}=893 \mathrm{~nm}$.
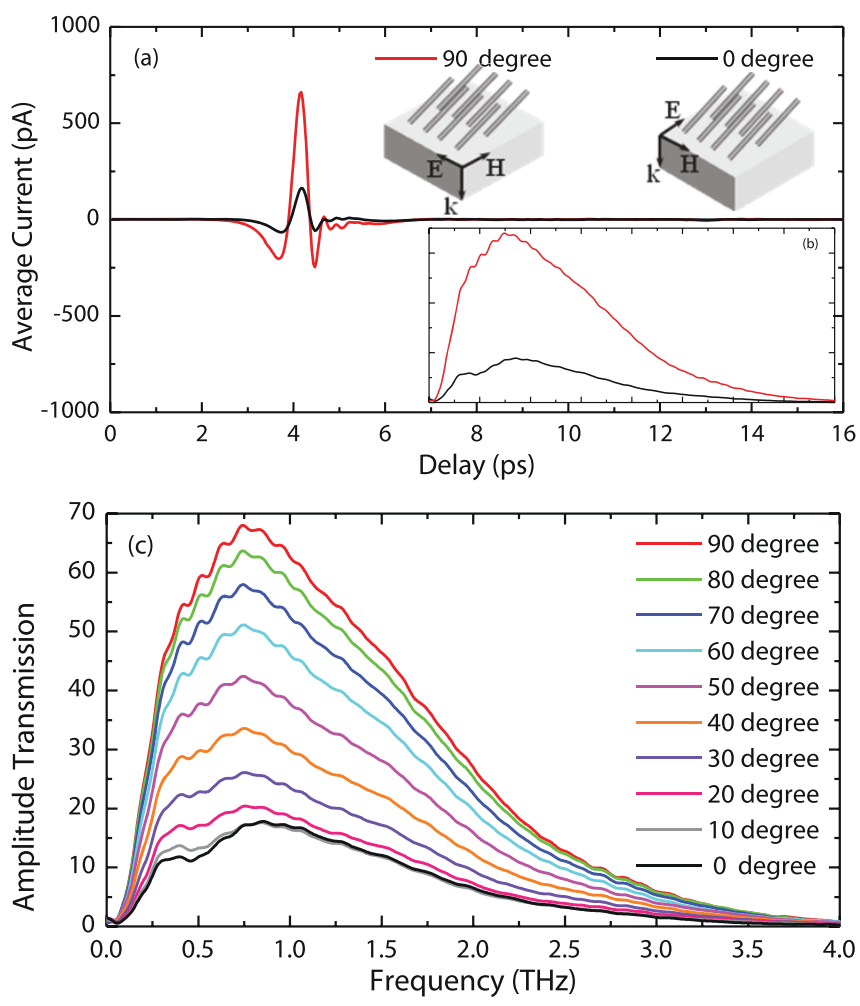

Figure $2 \mid$ THz-TDS measured results of uniform Ag nanorod arrays. (a) Time-domain pulses transmitted through the Ag nanorod array at angles of $0^{\circ}$ and $90^{\circ}$, respectively. (b) Corresponding Fourier-transformed spectra. (c) A complete set of THz-TDS spectra with the Ag nanorod array rotated about the normal to its surface at various angles.

contrast ratio in the amplitude transmission of THz pulses. Figure $2 \mathrm{c}$ shows a whole set of the polarization angle dependent transmission spectra. As the polarization angle increases from $0^{\circ}$ to $90^{\circ}$, the $\mathrm{THz}$ amplitude transmission becomes stronger.

Numerical simulations. Such a trend can be understood using numerical simulation by the finite-difference time-domain (FDTD) method (using the software package XFdtd (Remcom)). Figure $1 \mathrm{~b}$ shows the staggered base morphology of AgNRs derived from the SEM image. We treat the AgNR arrays as round-tipped cylinders oriented with a tilt angle of $72^{\circ}$ with respect to the surface normal and arranged in the plane of the substrate into a two-dimensional (2D) rhombic lattice with the lattice dimensions $L_{x}=250 \mathrm{~nm}$ and $L_{y}=893 \mathrm{~nm}$ as defined in Fig. 1b. Clearly this structure is an idealization of general features shown in Fig. 1a. The actual samples have rods connected at various areas and separated to various extents. The connectivity of the nanorods is evidenced by four-point probe measurements of the anisotropic resistance of AgNR sample (see Supporting Information). Thus, it is reasonable to further hypothesize that the rods are coupled by material connections that may be resistive or capacitive in nature. If the point of contact between rods is significantly smaller than the rod diameter, then the connection is resistive. If protuberances on rods are in very close proximity but not actually in contact, then the coupling is capacitive. Therefore, in the simulation we add resistive and capacitive connections to the $2 \mathrm{D}$ unit cell of the base morphology.

The simulations described here use $\sim 1$ ps Gaussian pulses centered at $\sim 1 \mathrm{THz}$. We chose a spatial grid cell size of $8 \mathrm{~nm}$ with an intent to resolve the base morphology while maintaining reasonable computational size. This choice results in a staircased spatial meshing of the rods with features that are at most $10^{-4} \times \lambda_{\text {min }}$, where $\lambda_{\text {min }}$ 
refers to the shortest wavelength contributing to the excitation spectrum. Spurious diffraction from curved material surfaces that are imperfectly realized on the discrete spatial grid may therefore be neglected. The $\sim 0.01 \mathrm{fs}$ time step that results from this choice according to the Courant stability condition ensures numerical stability for all frequencies and materials considered ${ }^{37}$. The size of the computational domain required was reduced by imposing periodic boundary conditions at the edges of the $893 \mathrm{~nm} \times 250 \mathrm{~nm}$ unit cell, which corresponds to a physical system that is infinite in the plane of the substrate. Drude behavior in the AgNRs of the base morphology is implemented according to the permittivity ${ }^{38}$ $\varepsilon(\omega)=1-\frac{\omega_{p}^{2}}{\omega(i \gamma+\omega)}$, where $\omega_{p}=1.37 \times 10^{16} \mathrm{rad} / \mathrm{s}$ is the plasma frequency and $\gamma=8.20 \times 10^{13} \mathrm{rad} / \mathrm{s}$ is the scattering frequency ${ }^{39-41}$. For resistive inter-rod coupling in the $y$-direction, $100 \mathrm{~nm}$ diameter cylindrical connectors with scattering frequency $\gamma$ increased by a factor of 16 are added at the rod tips. For resistive coupling in the $x$-direction, similar connectors with scattering frequency $\gamma$ increased by a factor of 3.5 are added at rod bases. An additional rod with a $20 \mathrm{~nm}$ thick dielectric spacer with a dielectric constant of 800 is added for capacitive coupling across rod tips in the $x$-direction. If the simplistic form of capacitance in terms of parallel plates of area $A$ separated by a dielectric with thickness $d$ is assumed, then the higher dielectric constant of the capacitive element can be reduced to correspond to that of air while keeping the capacitance constant if $A / d$ is increased by a factor of 800 . Taking $A$ to be the surface area of one of the rods and $d=1 \mathrm{~nm}$ constitutes one set of geometrical parameters that results in the same capacitance with unit dielectric constant. This combination of inter-rod coupling elements produces anisotropic high-pass filter behavior similar to that measured in our experiments (the computational morphology is shown in Supporting Information).

Comparison and analysis of simulated and experimental results. Figure $3 \mathrm{a}$ shows the simulated normalized amplitude transmission spectra of the AgNR array with varied incident polarization calculated by the FDTD method. The trends of the numerical spectra are in good agreement with the experimental results, as shown in Fig. 3b. In both experiment and simulation it is observed that transmission increases approximately linearly with frequency at angles greater than $30^{\circ}$, although this effect is more pronounced in experiment. At the polarization angles of $0^{\circ}$ and $15^{\circ}$, the transmittance remains almost unchanged with frequency. In all cases, transmission decays with decreased polarization angle. The majority of the transmission drop occurs between polarization angles $30^{\circ}$ and $75^{\circ}$. We consider the agreement between the simulation and experimental results shown in Fig. 3 to be limited by the constraint to morphologies with translational symmetry of such small lattice vectors. In other words, the investigation of connections within the smallest unit cell of the staggered base morphology is computationally convenient, but limits the accessible spatial extent of impedance-matched electrical pathways. With this limitation, the spatial confinement of uniformly damped oscillations in and on Drude materials is limited by the unit cell size. Longerrange impedance-matched electrical pathways are thought to be responsible for the non-monotonicity occurring in the normalized transmission spectra measured in our experiments at intermediate incident polarization angles as shown in Fig. 3b. We conclude that the nanorod shape and orientation produce effective anisotropic bulk conductivity to the $\mathrm{THz}$ radiation.

\section{Discussion}

Such polarization-dependent properties of the AgNRs can be used to design subwavelength plasmonic $\mathrm{THz}$ devices ${ }^{32,42}$. The AgNR film was lithographically patterned with a periodic array of subwavelength holes. The inset of Fig. 4a shows that the dimension of the
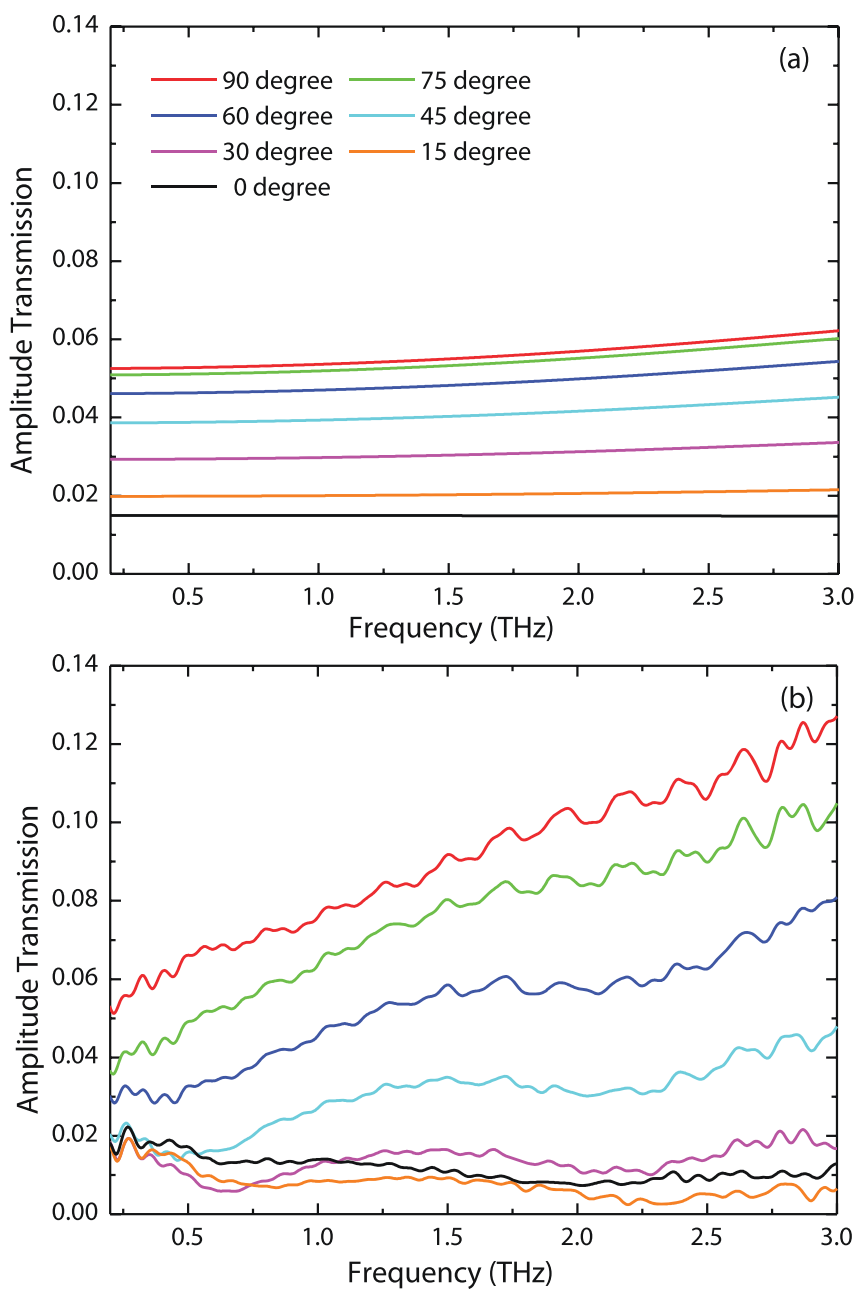

Figure 3 Normalized amplitude transmission spectra for various incident polarizations. (a) Simulated transmission spectra of the $\mathrm{Ag}$ nanorod array. (b) Experimental transmission spectra.

rectangular holes is $100 \mu \mathrm{m} \times 80 \mu \mathrm{m}$ and the lattice constant is $160 \mu \mathrm{m}$ in both $x$ and $y$ directions, thus the holes represent $31.25 \%$ of the surface area. The holes are smaller than the free-space resonance wavelength, i.e. $600 \mu \mathrm{m}(0.5 \mathrm{THz})$ in this case. The remaining surface area is uniformly covered with AgNRs. Two arrays are created with identical aperture dimensions, lattice constant, and thickness, but different nanorod orientations with respect to the rectangular holes. The first hole array (inset in Fig. 4a) has the direction of the nanorods tilted along the shorter $80 \mu \mathrm{m}$ side ( $\mathrm{x}$-axis) of the holes. We refer to this array as AgNR1. The second hole array (inset in Fig. 4b) has the axis of nanorods tilted along the longer, $100 \mu \mathrm{m}$ side ( $y$-axis) of the holes. We refer to this array as AgNR2. A linearly polarized $\mathrm{THz}$ wave was incident at normal incidence onto each of the structured surfaces, with the electric field either parallel or perpendicular to the shorter axis ( $x$-axis) of the holes. These two hybrid micro-/nano-structures show distinct $\mathrm{THz}$ response.

Figure 4a shows the $\mathrm{THz}$ transmission spectra obtained from hole array AgNR1. When the $\mathrm{THz}$ field is parallel to the long axis of the nanorods and the short axis of the holes in AgNR1, we observe the excitation of the fundamental $[0,1]$ SP resonance mode at the metallic nanorod-silicon interface at $0.5 \mathrm{THz}$. However, as the array AgNR1 is rotated by 90 degrees such that the $\mathrm{THz}$ field is parallel to the long axis of the holes, the SP resonance disappears. Thus, the excitation of the SP resonance in the hole array is strongly linked to the excitation of SPs in the individual nanorods. The electronic excitation in each nanorod becomes possible only when the incident 

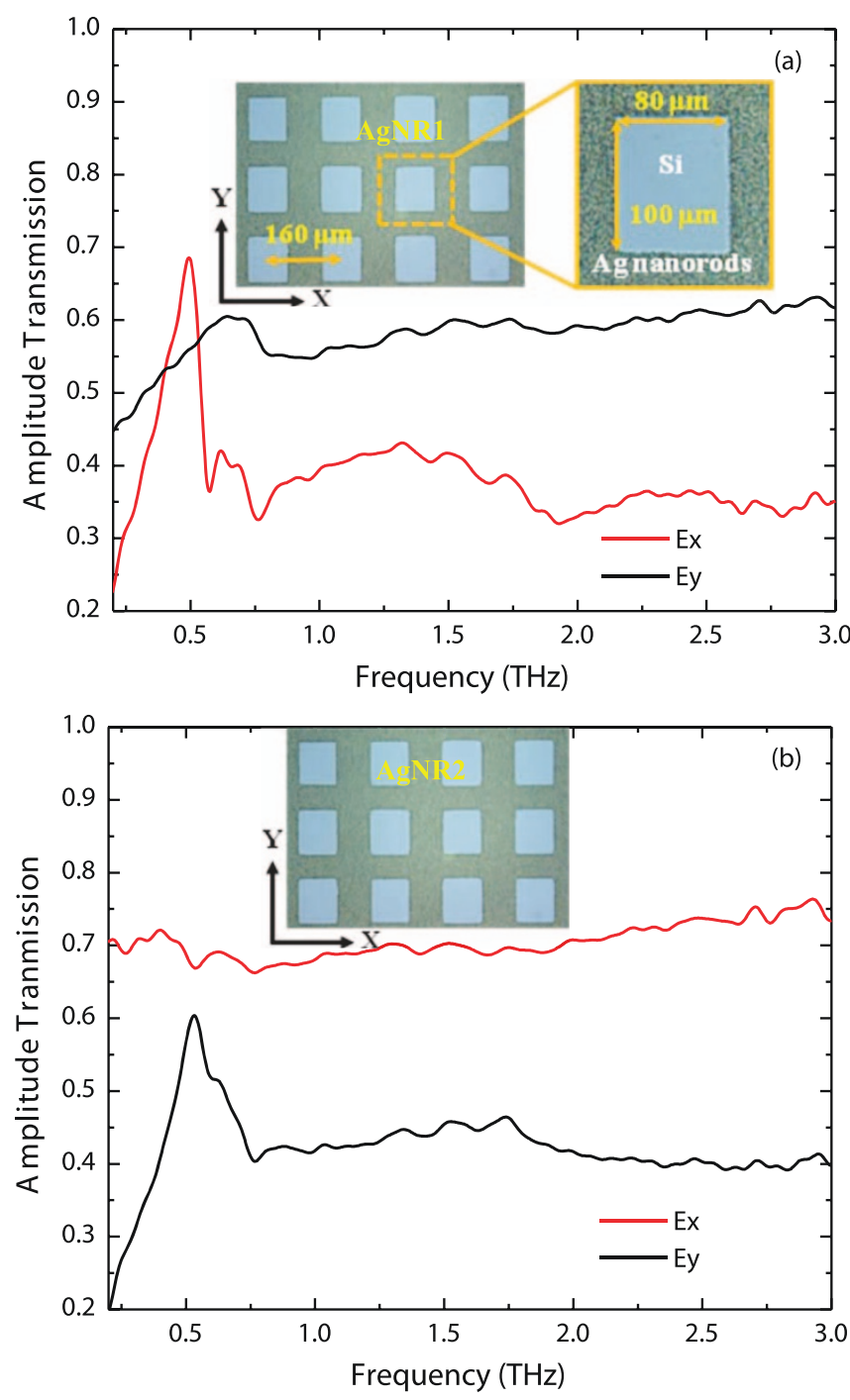

Figure $4 \mid$ Measured transmission through the AgNR arrays patterned with rectangular holes. Spectra of the measured amplitude transmission through both arrays are shown when the electric field is polarized either along the short axis or along the long axis of the holes. (a) AgNR1 is patterned with the nanorod projection along the shorter axis of the hole. (b) AgNR2 is patterned with the nanorod projection along the longer axis of the hole. The insets show optical microscope images of the $80 \mu \mathrm{m} \times$ $100 \mu \mathrm{m}$ rectangular hole arrays in the AgNR films.

$\mathrm{THz}$ field is aligned along the longer axis of the nanorods. In Fig. 4b, we observe a similar behavior in the second hole array AgNR2. The fundamental SP resonance mode is excited at $0.5 \mathrm{THz}$ when the electric field is aligned along the longer axis of the nanorods as well as of the holes. As this sample is rotated by 90 degrees, the resonance disappears since the $\mathrm{THz}$ waves do not couple to the nanorods and fail to excite the SPs. These unique polarization dependent properties are consistent with the idea of anisotropic effective $\mathrm{THz}$ conductivity in the Ag nanorod film, and clearly demonstrate an opportunity for developing new and more functional $\mathrm{THz}$ components and devices. The SP wave is excited in each nanorod. Since the nanorods are separated with a distance much smaller than the wavelength of the incident electromagnetic wave, the plasmon modes interact strongly with each other through their near fields giving rise to a collective resonance response. The local electric field intensity at the resonance could exceed the intensity of incident field by several orders of magnitude. Such intense fields can be exploited for enhanced spectroscopy, nonlinear processes, lithography, sensing and all related applications.

In summary, the polarization-dependent transmission of $\mathrm{THz}$ pulses through Ag nanorod array films was demonstrated both experimentally and numerically by varying the angle between the tilted nanorods and the electric field polarization. Resonant extraordinary transmission was measured through Ag nanorod films patterned with periodic arrays of rectangular subwavelength holes on a silicon substrate. The resulting spectroscopy points to the selective excitation of SPs according to the anisotropic conductivity of the nanorod film. Moreover, the results demonstrate that the anisotropic nanoscale surface morphology of the metallic rods gives rise to the material anisotropy observed at $\mathrm{THz}$ wavelengths $\sim 3$ orders of magnitude longer than the nanorod length scale. These unique material surfaces, used as lithographic substrates onto which subwavelength structures are patterned, provide a means for realizing novel $\mathrm{THz}$ polarization filtering, switching, tuning, sensing, and narrowband filtering devices. The nanorod plasmonic device also provides for a much increased surface area and intense fields in the interstitial space between the vertical rods that could be exploited for binding biomolecules that the nanorod hole array device would be able to sense easily.

\section{Methods}

THz-TDS measurements of the nanorod plasmonic geometries. A THz-TDS system was employed to characterize the nanorod samples. It consisted of a photoconductive-switch based transmitter and receiver and featured a frequencyindependent beam waist of $3.5 \mathrm{~mm}$, a useful bandwidth of 0.1 to $4.5 \mathrm{THz}(3 \mathrm{~mm}-$ $67 \mu \mathrm{m})$, and an amplitude signal to noise ratio $(\mathrm{S} / \mathrm{N})$ of $>10000: 1$. The short pulse terahertz wave propagated through the AgNRs and the silicon substrate $(640 \mu \mathrm{m}$ thick, n-type resistivity $\rho=12 \Omega \mathrm{cm}$ ) at normal incidence with the electric field parallel to the optical table. The transmitted THz signal was recorded in the time domain and subsequently converted to the frequency domain by Fourier transform.

1. Auston, D. H., Cheung, K. P., Valdmanis, J. A. \& Kleinman, D. A. Cherenkov radiation from femtosecond optical pulses in electro-optic media. Phys. Rev. Lett. 53, 1555 (1984).

2. Ketchen, M. B. et al. Generation of subpicosecond electrical pulses on coplanar transmission lines. Appl. Phys. Lett. 48, 751-753 (1986).

3. Zhang, X. C., Jin, Y. \& Ma, X. F. Coherent measurement of THz optical rectification from electro-optic crystals. Appl. Phys. Lett. 61, 2764-2766 (1992).

4. Grischkowsky, D., Keiding, S., Vanexter, M. \& Fattinger, C. Far-infrared timedomain spectroscopy with terahertz beams of dielectrics and semiconductors. J. Opt. Soc. Am. B 7, 2006-2015 (1990).

5. Hu, B. B. \& Nuss, M. C. Imaging with terahertz waves. Opt. Lett. 20, 1716-1718 (1995).

6. Ding, Y. J. Efficient generation of high-power quasi-single-cycle terahertz pulses from a single infrared beam in a second-order nonlinear medium. Opt. Lett. 29, 2650-2652 (2004).

7. Williams, B. S., Kumar, S., Hu, Q. \& Reno, J. L. Operation of terahertz quantumcascade lasers at $164 \mathrm{~K}$ in pulsed mode and at $117 \mathrm{~K}$ in continuous-wave mode. Opt. Express 13, 3331-3339 (2005).

8. Tonouchi, M. Cutting-edge terahertz technology. Nat. Photon. 1, 97-105 (2007).

9. Ferguson, B. \& Zhang, X. C. Materials for terahertz science and technology. Nat. Mater. 1, 26-33 (2002)

10. Mendis, R. \& Grischkowsky. Undistorted guided-wave propagation of subpicosecond terahertz pulses. Opt. Lett. 26, 846-848 (2001).

11. Averitt, R. D., Rodriguez, G., Siders, J. L. W., Trugman, S. A. \& Taylor, A. J. Conductivity artifacts in optical-pump $\mathrm{THz}$-probe measurements of $\mathrm{YBa}_{2} \mathrm{Cu}_{3} \mathrm{O}_{7}$. J. Opt. Soc. Am. B 17, 327-331 (2000).

12. Shan, J., Wang, F., Knoesel, E., Bonn, M. \& Heinz, T. F. Measurement of the frequency-dependent conductivity of sapphire. Phys. Rev. Lett. 90, 247401 (2003).

13. Markelz, A. G., Roitberg, A. \& Heilweil, E. J. Pulsed terahertz spectroscopy of DNA, bovine serum albumin and collagen between 0.1 and $2.0 \mathrm{THz}$. Chem. Phys. Lett. 320, 42-48 (2000).

14. Nagel, M. et al. Integrated THz technology for label-free genetic diagnostics. App. Phys. Lett. 80, 154-156 (2002).

15. Hanham, S. M. et al. Broadband terahertz plasmonic response of touching InSb disks. Adv. Mater. 24, 226-230 (2012).

16. Azad, A. K., Dai, J. M. \& Zhang, W. Transmission properties of terahertz pulses through subwavelength double split-ring resonators. Opt. Lett. 31, 634-636 (2006).

17. Singh, R., Azad, A. K., O’Hara, J. F., Taylor, A. J. \& Zhang, W. Effect of metal permittivity on resonant properties of terahertz metamaterials. Opt. Lett. 33, 1506-1508 (2008). 
18. Zhang, S. et al. Negative Refractive Index in Chiral Metamaterials. Phys. Rev. Lett. 102, 023901 (2009).

19. Chen, H. T. et al. A metamaterial solid-state terahertz phase modulator. Nat. Photon. 3, 148-151 (2009).

20. Tao, H. et al. A metamaterial absorber for the terahertz regime: design, fabrication and characterization. Opt. Express 16, 7181-7188 (2008).

21. Zhou, F., Bao, Y., Cao, W., Gu, J., Zhang, W. \& Sun, C. Hiding a realistic object using a broadband terahertz invisibility cloak. Sci. Rep. 1, 78 (2011).

22. Liang, D. et al. Robust large dimension terahertz cloaking. Adv. Mater. 24, 916 921 (2012).

23. O'Hara, J. F. et al. Thin-film sensing with planar terahertz metamaterials: sensitivity and limitations. Opt. Express 16, 1786-1795 (2008).

24. Chiam, S. Y. et al. Increased frequency shifts in high aspect ratio terahertz split ring resonators. Appl. Phys. Lett. 94, 064102 (2009).

25. Debus, C. \& Bolivar, P. H. Frequency selective surfaces for high sensitivity terahertz sensing. Appl. Phys. Lett. 91, 184102 (2007).

26. Singh, R. et al. Spiral-type terahertz antennas and the manifestation of the Mushiake principle. Opt. Express 17, 9971-9980 (2009).

27. Biagioni, P. et al. Nanoantennas for visible and infrared radiation. Rep. Prog. Phys. 75, 024402 (2012).

28. Razzari, L. et al. Extremely large extinction efficiency and field enhancement in terahertz resonant dipole nanoantennas. Opt. Express 19, 26088-26094 (2011).

29. Razzari, L. et al. Terahertz Dipole Nanoantenna Arrays: Resonance Characteristics Plasmonics. 8, 133-138 (2012). doi: 10.1007/s11468-012-9439-0.

30. Gu, J. et al. Active control of electromagnetically induced transparency analogue in terahertz metamaterials. Nat. Commun. 3, 1151 (2009).

31. Gu, J. et al. An active hybrid plasmonic metamaterial. Opt. Mater. Express 2, 1618-1623 (2012)

32. Qu, D., Grischkowsky, D. \& Zhang, W. Terahertz transmission properties of thin, subwavelength metallic hole arrays. Opt. Lett. 29, 896-898 (2004).

33. Chaney, S. B., Shanmukh, S., Zhao, Y.-P. \& Dluhy, R. A. Aligned silver nanorod arrays produce high sensitivity surface-enhanced Raman spectroscopy substrates. Appl. Phys. Lett. 87, 031908 (2005).

34. Driskell, J. D. et al. The use of aligned silver nanorod arrays prepared by oblique angle deposition as surface enhanced raman scattering substrates. J. Phys. Chem. C 112, 895-901 (2008)

35. Liu, Y. J., Chu, H. Y. \& Zhao, Y. P. Silver nanorod array substrates fabricated by oblique angle deposition: morphological, optical, and SERS characterizations. J. Phys. Chem. C 114, 8176-8183 (2010).

36. Zhang, W. Resonant terahertz transmission in plasmonic arrays of subwavelength holes. Eur. Phys. J. Appl. Phys. 43, 1-18 (2008).
37. Taflove, A. \& Hagness, S. C. Computational Electrodynamics: The FiniteDifference Time-Domain Method. 3rd ed. Artech House, (2005).

38. Jackson, J. D. Classical Electrodynamics. 3rd ed. John Wiley \& Sons: New York, (1998).

39. Kunz, K. S. \& Luebbers, R. J. The Finite Difference Time Domain Method For Electromagnetics. CRC Press: Boca Raton, (1993).

40. Ordal, M. A. et al. Optical properties of the metals $\mathrm{Al}, \mathrm{Co}, \mathrm{Cu}, \mathrm{Au}, \mathrm{Fe}, \mathrm{Pb}, \mathrm{Ni}, \mathrm{Pd}$, $\mathrm{Pt}, \mathrm{Ag}, \mathrm{Ti}$, and $\mathrm{W}$ in the infrared and far infrared. Appl. Opt. 22, 1099-1119 (1983).

41. Valentine, J. et al. Three-dimensional optical metamaterial with a negative refractive index. Nat. Mater. 455, 376-379 (2008).

42. Zhang, W. et al. Direct observation of a transition of a surface plasmon resonance from a photonic crystal effect. Phys. Rev. Lett. 98, 183901 (2007).

\section{Acknowledgments}

This work is partially supported by the National Science Foundation (Grant No. ECCS-1232081) and the National Science Foundation of China (Grant No. 61028011). CYS and YPZ thank the support from National Science Foundation (Grant No. ECCS-1029609).

\section{Author contributions}

Y.Z. and W.Z. proposed the plasmonic material with Ag nanorod arrays. T.E.L. completed numerical simulations. C.S. fabricated the Ag nanorod samples. W.C. fabricated the micro-structured patterns on $\mathrm{Ag}$ nanorod samples and performed all the measurements. W.C., T.E.L., R.S., J.F.O. and W.M.D. developed the analytical model and discussed the comparisons between simulations and experiments, W.C., R.S. and J.F.O. analyzed the measured data, Y.Z. and W.Z. supervised the theory and the measurements. All the authors discussed the results and contributed to the writing of the manuscript.

\section{Additional information}

Supplementary information accompanies this paper at http://www.nature.com/ scientificreports

Competing financial interests: The authors declare no competing financial interests.

License: This work is licensed under a Creative Commons

Attribution-NonCommercial-NoDerivs 3.0 Unported License. To view a copy of this license, visit http://creativecommons.org/licenses/by-nc-nd/3.0/

How to cite this article: Cao, W. et al. Tailoring terahertz plasmons with silver nanorod arrays. Sci. Rep. 3, 1766; DOI:10.1038/srep01766 (2013). 